\title{
Ácaros Oribátidos interesantes del País Vasco. Nuevas citas (Acari, Oribatida)
}

\author{
A. I. Caballero (*) y J. C. Iturrondobeitia $(*)$
}

\section{Introducción}

El presente trabajo es una selección de la parte faunística perteneciente al proyecto de investigación (AMB94-0178-CO2-02 ) realizado en la localidad de Mendata (Vizcaya) en un terreno parcelado para el cultivo de pino, y en el que se aplicaron diversos tratamientos con escorias procedentes de acerías y fertilizantes NPK. Los resultados que se presentan hacen referencia a especies que se han considerado de interés por ser primera cita para la Península Ibérica y/o el País Vasco, ampliándose así el conocimiento en la distribución de las mismas y aportando, en ocasiones, información taxonómica adicional de interés.

\section{Resultados}

Se han estudiado un total de 7.438 individuos pertenecientes a 10 especies que se citan por orden sistemático. Para cada una de ellas se aportan dimensiones, número de individuos estudiados y todo aquello que se ha creído conveniente destacar para dilucidar su posición taxonómica.

Liochthonius perfusorius Moritz, 1976

Dimensiones (longitud x anchura): (170 x 90) $\mu \mathrm{m}$. No individuos: 12.

Se trata de una nueva cita para la Península Ibérica. Las dimensiones de estos individuos entran dentro del rango $162.5-178.7 \mu \mathrm{m}$ de longitud por 85-102.5 $\mu \mathrm{m}$ de anchura dado por Moritz (1976a). Es probable que esta especie, en la Península Ibérica, haya sido confundida con otras dos muy próximas como son Liochthonius brevis (Michael,
1888) o bien L. hystricinus (Forsslund, 1942). Se diferencia fundamentalmente de la primera por la forma del sensilo, que en L. brevis es fusiforme con extremo distal redondeado o terminado en una única punta y el resto del sensilo con espinas que se disponen en dos filas mientras que en la especie que nos ocupa, la maza del sensilo está truncada y es biapical (Balogh \& Mahunka, 1983). De L. hystricinus se diferencia, además de por el sensilo, por la longitud de las setas notogastrales siendo las de esta especie sensiblemente más largas que las de $L$. perfusorius.

Mixochthonius pilososetosus (Forsslund, 1942)

Dimensiones (longitud x anchura): (180 x 80) $\mu \mathrm{m}$.

No individuos: 3 .

Primera cita para el País Vasco y recientemente ha sido citada en Navarra en un trabajo de tesis doctoral (Jiménez, 1998) como nueva cita para la Península Ibérica. Para su identificación nos hemos basado en los trabajos de Moritz (1976b) y Balogh \& Mahunka (1983). Hay que decir que la única diferencia encontrada hace referencia al tamaño de los individuos, ya que las dimensiones que dan dichos autores para esta especie son más grandes (195-230 $\mu \mathrm{m}$ de longitud por 90-102.5 $\mu \mathrm{m}$ de anchura). Se le asigna una distribución holártica (en el área del Mediterráneo-Occidental sólo era conocida del Este, Subías \& Gil-Martín, 1997) pero no se cita en la Península Ibérica. Se trata de una especie muy difícil de localizar en las muestras debido a que se confunde fácilmente con formas inmaduras por su pequeño tamaño y sobre todo por su tegumento que es prácticamente transparente. La maza del sensilo es fusifome y ciliada, todas las setas, tanto del prodorso como notogáster, son largas y barbuladas siendo estas barbulaciones a su vez largas.

* Dpto. Zoología y Dinámica Celular Animal. Facultad de Ciencias. Universidad del País Vasco. B o Sarriena s/n. E-48940 Leioa (Vizcaya). 
Verachthonius laticeps (Strenzke, 1951)

Dimensiones (longitud x anchura): (190 x 90-100) $\mu \mathrm{m}$. No individuos: 282

Es la primera vez que se cita para el País Vasco. El tamaño de los individuos estudiados entra dentro del rango de valores aportado por Moritz, 1976a (182.5-207 x 95-110) $\mu \mathrm{m}$, así como la descripción de los caracteres morfológicos más importantes para su identificación. La placa suprapleural $S p C$ aparece fusionada a la placa $N a$ y sólo se distingue claramente la placa $S p E$. Maza del sensilo fusiforme con barbulaciones cortas finas y dispuestas en hileras longitudinales de 8 ó 10 espinas por hilera (Balogh \& Mahunka, 1983). Distribución paleártica y dentro del Mediterráneo-Occidental: Península Ibérica y Este (Subías \& Gil-Martín, 1997).

Steganacarus michaeli Bernini y Avanzati, 1987

Dimensiones (longitud $\mathrm{x}$ anchura): notogáster (570-900 $\mathrm{x}$ 400-660) $\mu \mathrm{m}+$ aspis $(270-430 \mathrm{x} 140-260) \mu \mathrm{m}$. No individuos: 21.

Primera cita para la Península Ibérica. Se trata de una especie de distribución paleártica-occidental (Subías \& Gil-Martín, 1997). Hasta ahora esta especie, a pesar de haber aparecido en algunas de las muestras de los diferentes proyectos en los que se ha trabajado, había sido confundida (Saloña, com. pers., 1995) con otra muy próxima a ella, Steganacarus (Steganacarus) magnus (Nicolet, 1855). En los trabajos de Bernini \& Avanzati, 1987 y 1988, quedan recogidas y perfectamente descritas las diferencias entre ambas especies y las variaciones en la última respecto a tamaños y formas.

Microtritia minima (Berlese, 1904)

Dimensiones (longitud x anchura): (260-290 x 160-180) $\mu \mathrm{m}$. $\mathrm{N}^{\mathrm{o}}$ individuos: 5.366 .

Fue citada en la Península Ibérica por primera vez por Subías (1977) como Microtritia xilofila pero después de haber estudiado su material y compararlo tanto con el material aparecido en el País Vasco hasta ahora citado como $M$. xilofila y teniendo en cuenta la revisión de Niedbala (1993) sobre el material tipo de la especie de Berlese (1904) (cuyas dimensiones son $320 \mu \mathrm{m}$ de longitud por $190 \mu \mathrm{m}$ de anchura), se confirma el criterio de Niedbala de sinonimizar Microtritia xilofila con Microtritia minima.

Damaeus (Damaeus) maximus Mihelcic, 1957

Dimensiones (longitud x anchura): (1010-1100 x 630750) $\mu \mathrm{m}$. $\mathrm{N}^{\mathrm{o}}$ individuos: 24

Nueva cita para el País Vasco, si bien ha sido previamente citado por otros autores en otras loca- lidades de la Península Ibérica (Pérez-Íñigo, 1997). Se trata de una especie de talla muy grande. Es relevante el comportamiento reproductor de la especie (Caballero \& Iturrondobeitia, 1999).

\section{Lauroppia fallax (Paoli, 1908)}

Dimensiones (longitud $\mathrm{x}$ anchura): $(250 \mathrm{x} 125) \mu \mathrm{m}$. No individuos: 1

En todo el trabajo sólo se ha encontrado un individuo hembra cuya descripción coincide con el trabajo de revisión de Arillo \& Subías (1996). Hasta hace poco no había sido hallada en el País Vasco. Sin embargo, Basáñez (1997) (trabajo inédito de tesis de licenciatura) encuentra cuatro individuos en un pinar joven de Guipúzcoa cuyas dimensiones oscilan entre los siguientes valores: longitud: $235-280 \mu \mathrm{m}$; anchura: 120-140 $\mu \mathrm{m}$. El único ejemplar que aparece en este trabajo se encuentra dentro de este rango, pero hay que señalar que son tamaños ligeramente inferiores a los que se dan por otros autores españoles para individuos encontrados en la Península Ibérica -Pérez-Íñigo (1971), Subías \& Arillo (1991) y Arillo \& Subías (1996) — cuyos valores oscilan en el rango 270-365 $\mu \mathrm{m}$ de longitud por $135-200 \mu \mathrm{m}$ de anchura. Estos son mucho menores que los que dan autores europeos Paoli (1908), Bruckner (1995), que encuentran ejemplares dentro de un rango mucho más amplio hasta llegar a las $415 \mu \mathrm{m}$ de longitud por $212 \mu \mathrm{m}$ de anchura.

\section{Ramusella (Insculptoppia) fusiformis (Wallwork, 1961)}

Dimensiones (longitud x anchura): (290-340 x 130-170) $\mu \mathrm{m}$. $\mathrm{N}^{\mathrm{o}}$ individuos: 22 .

Primera cita para la Península Ibérica. La especie Oppia soror var. fusiformis (Wallwork, 1961) posteriormente Oppia fusiformis (Wallwork, 1964) queda asignada dentro del género Ramusella (Insculptoppia) Subías, 1980. La característica fundamental de este subgénero son las setas rostrales (ro), uniformemente curvadas, sin acodamiento, y convergiendo sus extremos. Para el caso concreto de la especie $R$. (I.) fusiformis hay que destacar la presencia de unas pequeñas setas ta así como la longitud de las setas interlamelares (in), características morfológicas que aparecen en nuestro material. En cuanto a las dimensiones de los individuos, Wallwork (1961) da un rango de comprendido entre 241.5 y $291.1 \mu \mathrm{m}$ de longitud por 140 $\mu \mathrm{m}$ de anchura, es decir, algo menores que los individuos que aparecen en nuestro trabajo. Creemos que esta especie en algunas ocasiones ha podido ser confundida con $R$. (Ramusella) puerto- 
monttensis Hammer, 1962. a pesar de la ausencia de setas $t a$ en esta especie.

\section{Ramusella (Ramusella) clavipectinata (Michael,} 1885)

Dimensiones (longitud $\mathrm{x}$ anchura): (244-350 x 125-200) $\mu \mathrm{m} ;(250-360$ x 135-200) $\mu \mathrm{m}$. № de individuos: 1.215 .

En el trabajo de Arillo y Subías (1995) se sinonimiza la especie $R$. (R.) assimilis (Mihelcic, 1956) con $R$. (R.) clavipectinata (Michael, 1885), y se pasa a nivel de subespecie a $R$. (R.) clavipectinata assimiloides Subías et Rodríguez, 1987, si bien esta última con ciertas dudas ya que los autores reflejan la posibilidad de que los individuos estudiados sean, en realidad, morfotipos de la misma especie debido a la variabilidad de los caracteres morfológicos que las diferencian. Estos son: forma de la prominencia rostral, ramas del sensilo y orientación del discidio. En este trabajo, si bien se ha utilizado ese criterio a la hora de diferenciar las especies, hay que decir que no es fácil determinar siempre a qué grupo pertenecen debido a la ya mencionada variabilidad de caracteres, de tal manera que como ya mencionaba Arillo (1996) en su tesis doctoral propone la sinonimia, quedando una sola especie Ramusella (Ramusella) clavipectinata sensu lato en la que se incluirían ambos morfotipos.

\section{Ceratozetes peritus Grandjean, 1951}

Dimensiones (longitud x anchura): (510-590 x 300-410) $\mu \mathrm{m} . \mathrm{N}^{\mathrm{o}}$ de individuos: 492 .

Los individuos que habían sido identificados en el País Vasco como Ceratozetes gracilis (Michael, 1884) pertenecen a la especie Ceratozetes peritus Grandjean, 1951. Se ha tenido ocasión de estudiar el material tipo de $C$. gracilis de la colección de Michael en el British Museum de Londres, y se ha comprobado, tal y como apunta Behan-Pelletier
(1984), que son varias las características morfológicas que separan a ambas especies además de la estriación de las lamelas que indica Pérez-Íñigo (1993) en la clave de identificación de las especies del género Ceratozetes de la Península Ibérica e Islas Baleares. El compendio de características utilizadas para la identificación de todos los individuos que aparecen en este trabajo se resume en la Tabla 1. Todo el material ha resultado pertenecer a la especie C. peritus, y además, revisando el material de la colección depositada en el laboratorio de Zoología de esta Universidad, hay que considerar que, hasta la fecha, la especie $C$. gracilis no ha aparecido en el País Vasco.

\section{AGRADECIMIENTOS}

Trabajo subvencionado por el proyecto $n^{\circ}$ ref.: AMB940178-CO2-02 y por una beca predoctoral del Ministerio de Educación y Cultura

\section{Referencias}

AriLlo, A.,1996. Estudio faunístico y Taxonómico de los Oppidos del sur de España (Acari, Oribatida, Oppiidae). Memoria de Tesis Doctoral. Universidad Complutense de Madrid, Fac. de Ciencias Biológicas, Dpto. de Biología Animal I (Entomología). 322 pp. (inédita).

Arillo, A. \& Subías, L.S., 1995. Los Multioppiinae del sur de España II. El género Ramusella con descripción de una nueva especie (Acari, Oribatida, Oppiidae). Graellsia, 51: 45-53.

AriLlo, A. \& SuBÍAs, L.S., 1996. Los Oppiellinae en el sur de España: géneros Lauroppia, Moritzoppia, Neotrichoppia (Confinoppia) y Oppiella. Descripción de Lauroppia baetica sp. n. (Acari, Oribatida, Oppiidae). Miscel'lània Zoològica, 19(2): 53-66.

Tabla 1.- Diagnosis diferencial entre las especies C. peritus y C. gracilis.

Table 1.- Differential diagnosis between Ceratozetes peritus and C. gracilis.

\begin{tabular}{|c|c|c|}
\hline & Ceratozetes peritus Grandjean, 1951 & Ceratozetes gracilis (Michael, 1884) \\
\hline Rostro & $\begin{array}{l}2 \text { dientes laterales con incisión } \\
\text { (no muy profunda) ancha entre ellos }\end{array}$ & $\begin{array}{l}2 \text { dientes laterales con profunda } \\
\text { incisión entre ambos }\end{array}$ \\
\hline Botridio (escama psdm) & convexa & cóncava \\
\hline Setas interlamelares (in) & Alcanzan cúspides lamelares & No alcanzan cúspides lamelares \\
\hline Lamela & Sin estriación, lisas & Con estriación longitudinal \\
\hline
\end{tabular}


Balogh, J. \& MahunKa, S., 1983. Primitive Oribatids of the Palaearctic Region. In: J. Balogh \& S. Mahunka (eds.). The soil mites of the World. Elsevier Science Publishers B. Amsterdam. Vol I. 372 pp.

BASÁÑEZ, A., 1997. Contribución al conocimiento de los Oribátidos (Acari) de Gipúzkoa. Memoria de Tesis de Licenciatura. Dpto. Zoología y Dinámica Celular Animal. Fac. Ciencias. U.P.V.-E.H.U. 69 pp. (inédita).

Behan-Pelletier, V.M., 1984. Ceratozetes (Acari: Ceratozetidae) of Canada and Alaska. Canadian Entomologist, 116: 1449-1517.

Bernini, F. \& Avanzati, A.M., 1987. Notulae Oribatologicae XLI. Some new Steganacarus species belonging to the magnus group (Acarida, Oribatida). International Journal of Acarology, 13(3): 165-176.

Bernini, F. \& Avanzati, A.M., 1988. Taxonomic revision of Steganacarus (Steganacarus) magnus (Nicolet, 1855) (Acarida, Oribatida). Journal of Natural History, 22: 435- 464.

BRUCKNER, A., 1995. Cave-dwelling oribatid mites (Acarina, Cryptostigmata) from East Austria. Verhandlungen der Zoologisch-Botanischen Gesellschaft in Österreich, 132: 81-107.

Caballero, A.I. \& Iturrondobeitia, J.C., 1999. Observaciones en la biología reproductiva de Damaeus maximus Mihelcic, 1957 (Acari, Oribatei). Boletín de la Asociación española de Entomología, 23(1-2): 223-230.

JIMÉNEZ, A., 1998. Alteración, evolución temporal y recuperación, tras los incendios forestales, de la Comunidad Acarológica en un medio forestal. Memoria de Tesis Doctoral. Dpto. Zoología y Ecología. Facultad de Ciencias. Universidad de Navarra. Pamplona. 390 pp. (inédita).

Moritz, M., 1976a. Revision der Europäischen Gattungen und Arten der Familie Brachochthoniidae (Acari, Oribatei). Mitteilungen aus dem Zoologischen Museum in Berlin, 52(1): 29-136.

Moritz, M., 1976b. Revision der Europäischen Gattungen und Arten der Familie Brachochthoniidae (Acari, Oribatei). Mitteilungen aus dem Zoologischen Museum in Berlin, 52(2): 227-319.

NiedBala, W., 1993. Revision of Oribatid mites from Berlese's Collection. III. Redescription of species of Mesoplophoroidea and Euphthiracaroidea (Acari, Oribatida). Genus (Wroclaw), 4(1): 41-58.

Paoli, G., 1908. Monografia del genere Dameosoma Berlese e generi affini. Redia, 5: 31-91.
PÉrez-ÍÑIgO, C., 1971. Ácaros Oribátidos de suelos de España peninsular e Islas Baleares. III. Eos (Madrid), 46: 263-350.

PÉreZ-ÍÑIGO, C., 1993. Acari, Oribatei, Poronota. En: Ramos, M.A. et al. (eds.). Fauna Ibérica, vol. 3. Museo Nacional de Ciencias Naturales, CSIC. Madrid. 320 pp.

PÉrez-ÍÑIGO, C., 1997. Acari, Oribatei, Gymnonota I. En: Ramos, M.A. et al. (eds.). Fauna Ibérica, vol. 9. Museo Nacional de Ciencias Naturales, CSIC. Madrid. 374 pp.

SubÍAs, L.S., 1977. Taxonomía y Ecología de los Oribátidos Saxícolas y Arborícolas de la Sierra de Guadarrama (Acarida, Oribatida). Trabajo $\mathrm{n}^{\circ} 24$ Cátedra de Artrópodos, Departamento de Zoología, Facultad de Biología, Universidad Complutense de Madrid. 379 pp.

SuBíAs, L.S., 1980. Oppiidae del complejo "clavipectinata-insculpta" (Acarida, Oribatida). Eos (Madrid), 54: 281-313.

SubíAs, L.S. \& ARILlo, A., 1991. Los Oppiidae Grandjean, 1951 (Acari, Oribatida) de Madeira. Vieraea, 20: 39-52.

SubíAs, L.S. \& Gil-Martín, J., 1997. Systematic and biogeographic checklist of oribatids from western Mediterranean (Acari, Oribatida). Annali del Museo di Storia Naturale "Giacomo Doria”, 91: 459-498.

SuBíAs, L.S. \& RodríGuEz, P., 1987. Oppiidae (Acari, Oribatida) de los sabinares (Juniperus thurifera) de España I. Ramusella s. str. Hammer y Ramusella (Rectoppia) Subías. Eos (Madrid), 63: 301-314.

WALLwORK, J.A., 1961. The Oribatei from Ghana. VII Members of the "family" Eremaeidae Willmann ( $2^{\text {nd }}$ series). The genus Oppia Koch. Acarologia, 3(4): 637-658.

WAllwork, J.A., 1964. Some Oribatei (Acari: Cryptostigmata) from Tchad (1st. series). Revue de Zoologie et Botanique Africaines, 70(3-4): 353-385. 